\section{BRAZIULIAN JOURNAL}

OF MEDICAL AND BIOLOGICAL RESHARCH

www.bjournal.com.br
ISSN 0100-879X

Volume 43 (9) 812-913 September 2010

BIOMEDICAL SCIENCES

AND

CLINICAL INVESTIGATION

Braz J Med Biol Res, September 2010, Volume 43(9) 874-882

doi: 10.1590/S0100-879X2010007500083

Protective effect of maternal prenatal melatonin administration on rat pups born to mothers submitted to constant light during gestation

C.D. Cisternas, M.V. Compagnucci, N.R. Conti, R.H. Ponce and N.T. Vermouth

The Brazilian Journal of Medical and Biological Research is partially financed by

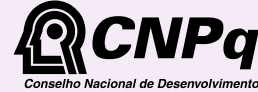

Ministério da Ciência e Tecnologia

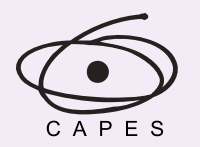

Ministério da Educação

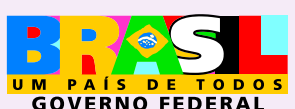

GOVERNO FEDERAL
TFAPESP

Institutional S ponsors

Hotsite of proteomics metabolomics developped by:$$
\text { อ }
$$

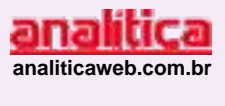

Thermo
sctiturfic 


\title{
Protective effect of maternal prenatal melatonin administration on rat pups born to mothers submitted to constant light during gestation
}

\author{
C.D. Cisternas ${ }^{1 *}$, M.V. Compagnucci ${ }^{1 *}$, N.R. Conti ${ }^{1}$, \\ R.H. Ponce ${ }^{1,2}$ and N.T. Vermouth ${ }^{1,2}$
}

${ }^{1}$ Facultad de Ciencias Médicas, and ${ }^{2}$ Facultad de Odontología, Universidad Nacional de Córdoba, Córdoba, Argentina

\begin{abstract}
We studied the effects of adverse conditions such as constant light (LL) on the circadian rhythm of malate (MDH, EC 1.1.1.37) and lactate (LDH, EC 1.1.1.27) dehydrogenase activities of the testes of male Wistar rats on postnatal day 28 (PN28), anxietylike behavior (elevated plus-maze test) at PN60 and sexual behavior at PN120. The rats were assigned to mother groups on day 10 of pregnancy: control (12-h light/dark), LL (light from day 10 to 21 of pregnancy), and LL+Mel (LL and sc injection to the mothers of a daily dose of melatonin, $1 \mathrm{mg} / \mathrm{kg}$ body weight at circadian time 12, from day 17 to 21 of pregnancy). LL offspring did not show circadian rhythms of $\mathrm{MDH}(\mathrm{N}=62)$ and $\mathrm{LDH}(\mathrm{N}=63)$ activities (cosinor and ANOVA-LSD Fisher). They presented a $44.7 \%$ decrease in open-arm entries and a $67.9 \%$ decrease in time (plus-maze test, $\mathrm{N}=15, \mathrm{P}<0.001$, Mann-Whitney $\mathrm{U}$-test and Kruskal-Wallis test), an increase in mounting (94.4\%), intromission (94.5\%) and ejaculation (56.6\%) latencies $(\mathrm{N}=12, \mathrm{P}$ $<0.01$, Mann-Whitney U-test and Kruskal-Wallis test) and lower numbers of these events (61, 59 and $73 \%$, respectively; $\mathrm{P}<$ $0.01, \mathrm{~N}=12$ ) compared to controls. The offspring of the $\mathrm{LL}+$ Mel group presented MDH and LDH circadian rhythms ( $<0.05$, $\mathrm{N}=50$, cosinor and ANOVA-LSD Fisher), anxiety-like and sexual behaviors similar to control. These findings supported the importance of the melatonin signal and provide evidence for the protective effects of hormones on maternal programming during gestation. This protective action of melatonin is probably related to its entrainment capacity, favoring internal coupling of the fetal multioscillatory system.
\end{abstract}

Key words: Pineal hormone; Constant light; Entrainment; Copulatory behavior; Anxiety-like behavior

\section{Introduction}

Melatonin is a multifunctional indolamine synthesized and secreted by the pineal gland and other organs (1). In rodents, the pattern of melatonin secretion is controlled by an endogenous circadian timing system and conveys information about the light-dark cycle to the organism, thus organizing its seasonal and circadian rhythms (1). Melatonin also provides significant beneficial protection due to its powerful antioxidant effects and immunomodulatory activity against cellular degeneration associated with aging and adverse conditions (2). The antioxidant properties of melatonin are related to its neuroprotective activity (3). Baydas et al. (4) showed a reduction of neural tissue lipid peroxidation in rat brain caused by constant light (LL) protecting both neurons and glial cells from free radicals.

Melatonin, one of the few maternal hormones crossing the placenta without being altered, may play an important role in the regulation of fetal daily rhythms (5-7). The fetus, whose pineal gland does not synthesize melatonin, is exposed to the maternal melatonin rhythm that begins to increase from the 12th day of gestation in rats (8). Hormone binding sites are identified in the suprachiasmatic nucleus (SCN) of a fetus from the 18th day of gestation (9). This melatonin path, which mediates interactions between maternal and fetal physiological functions, has been explored mostly in fetal and neonatal circadian rhythms (10-12).

In mammals, the SCN acting at the top of a hierarchical

Correspondence: N.T. Vermouth, Cátedra de Bioquímica y Biología Molecular, Facultad de Ciencias Médicas, Universidad Nacional de Córdoba, Pabellón Argentina, Haya de la Torre, s/n, 2do. Piso, Ciudad Universitaria, X5000 Córdoba, Argentina.

E-mail:nvermo@odo.unc.edu.ar

*These authors contributed equally to this study.

Received April 13, 2010. Accepted July 28, 2010. Available online August 27, 2010. Published September $13,2010$. 
system coordinates the timing of the endocrine system and reproductive behavior (13). During the fetal and neonatal periods, a maternal program regulates the development of circadian system $(14,15)$. The external environment of the fetus is provided by its mother. Rodent pups, maintained under constant light or darkness from birth, exhibit freerunning oscillations synchronized by their mothers $(16,17)$. Lesion of the maternal SCN during early gestation prevents maternal synchronization of pup rhythms (18). The removal of the pineal gland or of both superior cervical ganglia before the 11th day of gestation also prevents maternal synchronization of testicular malate dehydrogenase (MDH) rhythm and daily drinking behavior in the offspring $(11,12)$. These disruptions of circadian rhythms in the offspring are prevented by administration of melatonin to the mother during the last period of gestation $(7,12)$.

It is known that chronic exposure of adult rats to constant light induces suppression of the circadian system (19). The prenatal environment has a strong influence on the development of organisms. Exposure to adverse conditions during fetal life has been recognized as a disruptive factor for individual development and can cause short- and long-term effects on neuroendocrine and behavioral responses (20). In the present study, we applied LL as an adverse condition to pregnant rats and examined the circadian rhythms of lactate dehydrogenase (LDH) and $\mathrm{MDH}$, both testicular enzymes of daily variation $(21,22)$. On the other hand, stressful treatment of pregnant rats has been shown to cause remarkable changes in offspring physiology such as anxiety-like (23) and sexual behavior (24).

Since the SCN, one of the components of the circadian system, acts at the top of the hierarchical system, we expected that melatonin administration to pregnant rats exposed to LL would prevent postnatal alterations in the daily activity of testicular enzymes as well as in the behavioral patterns of rat pups. For this purpose, we checked the circadian rhythms of testicular MDH (EC 1.1.1.37) and LDH (EC 1.1.1.27) enzymes in young pups and assessed the patterns of anxiety-like and sexual behavior in adult offspring.

\section{Material and Methods}

Three-month-old Wistar rats maintained at $23 \pm 1^{\circ} \mathrm{C}$ with laboratory chow and water ad libitum and on a schedule of 12-h light:12-h dark (lights on from 7:00 am to 7:00 pm) were used. The time of activity onset under dark conditions was defined as circadian time 12 (CT12). Estrous cycles were determined by a daily examination of vaginal smears and animals were mated on the night of ovulation. The onset of pregnancy was confirmed by the presence of spermatozoa in vaginal smears on the following morning, considered to be day 0 of gestation. Pups from each group remained with their biological mothers after birth and during lactation. After delivery, on postnatal day 3 (PN3), litters were reduced to eight animals per mother and pups were weaned on day 21 .
The rats were experimentally naive.

All experimental procedures were approved by the local Bioethics Committee of the Facultad de Odontología, Universidad Nacional de Córdoba, Argentina, and followed the NIH Guidelines for the Care and Use of Laboratory Animals. We made all possible efforts to minimize both the suffering and the number of animals used.

\section{Animal groups}

The following groups were established on the 10th day of gestation.

Control group. Pups born to 15 mothers submitted to a schedule of 12-h light:12-h dark until the 21st day of gestation and injected subcutaneously with a daily dose of ethanol/saline solution from the 17th to the 21 st day of pregnancy at CT12.

LL group. Pups born to a group of 15 mothers submitted to constant cool white light (100 lux) from the 10th to the 21st day of gestation and injected subcutaneously with a daily dose of ethanol/saline solution from the 17 th to the 21 st day of pregnancy as described for the control group.

$L L+M e l$ group. Pups born to 15 mothers submitted to constant light during pregnancy, as described for the LL group, and injected subcutaneously $(7,12)$ with a daily dose of melatonin (Mel, $1 \mathrm{mg} / \mathrm{kg}$ body weight, CT12) from the 17 th to the 21st day of pregnancy, a period considered to be adequate to cause entrainment, as previously reported $(6,12)$. LL causes a 6 -fold decrease in mean plasma melatonin concentration in adult rats (20) and, under a light-dark cycle, the circulating plasma levels increase significantly from day 15 to reach the highest values on gestational day 21 (8). Thus, melatonin was administered during the last period of pregnancy in order to restore the melatonin signal. The pineal hormone melatonin was dissolved in $0.05 \mathrm{~mL}$ ethanol and then diluted up to 10 $\mathrm{mL}$ with $0.9 \%$ saline. The final concentration of ethanol was $0.5 \%$. Melatonin was purchased from Sigma (USA).

\section{Testicular circadian rhythms of the offspring}

In order to study the early effects of prenatal treatments, we assessed daily oscillations in testicular MDH and LDH, enzymes involved in energy metabolism. A set of mothers from the control, LL, and LL+Mel groups was exposed to constant darkness (DD) from gestational day 21. After delivery, mothers and litters were maintained in DD until sacrifice to avoid their synchronization with the light-dark cycle. The animals were manipulated under dim red light at different times. On the weaning day (PN21), male pups were taken randomly from different mothers and allocated to time groups of 5-8 animals each. At PN28, pups were killed in groups by decapitation every 3-4 h during a 24-h period. After sacrifice, the testes were immediately removed, dissected and kept at $-24^{\circ} \mathrm{C}$ until enzyme determination. The tissue without the capsule was suspended in 24 parts of distilled water (1:25, w:v) and homogenized with a tissue disruptor. Suspensions were centrifuged at $13,000 \mathrm{~g}$ for $30 \mathrm{~min}$ at $4^{\circ} \mathrm{C}$ and supernatants were used for MDH and LDH assay. 
MDH assay. Testicular MDH (EC 1.1.1.37) activity was determined by the method of Ochoa (25) using $1 \mathrm{mM}$ oxaloacetate as substrate. The assay was performed at $37^{\circ} \mathrm{C}$. Changes in absorbance at $340 \mathrm{~nm}\left(\mathrm{E}_{340}\right)$ were recorded for a 3-min period. One unit of enzyme is the amount producing a $\Delta \mathrm{E}_{340}$ of 2.07 per min, which corresponds to the oxidation of $1 \mu \mathrm{mol} \mathrm{NADH}$.

LDH assay. LDH (EC 1.1.1.27) activity was determined using $0.5 \mathrm{mM} \mathrm{Na}$ pyruvate as substrate according to the method of Wroblesky and LaDue (26). The assay conditions were similar to those described for $\mathrm{MDH}$ determination. One unit of enzyme is the amount producing a $\Delta \mathrm{E}_{340}$ of 2.07 per min, which corresponds to the oxidation of $1 \mu \mathrm{mol} \mathrm{NADH}$.

We have previously determined a circadian fluctuation of protein content in testis extracts (21). Given that this is an extra source of variation in terms of specific enzyme activity (units/mg protein), a more reliable index for enzyme activity is obtained by reporting the results as units per $g$ of wet tissue.

\section{Behavioral tests}

In order to study the long-term effects of prenatal treatments, male offspring were submitted to the elevated plusmaze test at PN60 as well as to the copulatory behavior test at PN120. We used animals born and maintained in DD from control, LL and LL+Mel groups.

Elevated plus-maze test. The elevated plus-maze test is a paradigm widely used to investigate anxiety-related behavior that has been validated in rats (27). It is based on the testinduced conflict between the aversion of being exposed in an open-elevated platform and the motivation to explore the new environment. As a consequence, the less anxious the individuals, the more the exploration into the open arms. The platform was raised $50 \mathrm{~cm}$ above the floor and consisted of two open arms $(10 \times 50 \mathrm{~cm})$ and two enclosed arms $(10 \times 50$ $x 45 \mathrm{~cm}$ high walls) extending from a central platform (10 $\mathrm{x}$ $10 \mathrm{~cm}$ ). The animals were handled daily by the same experimenter for at least 15 days prior to testing. The experimental room was lit by a $60-\mathrm{W}$ bulb placed $1.75 \mathrm{~m}$ above the central square of the maze (22 lux in the central square of the maze). Each animal group was allowed to rest in the experiment room $1 \mathrm{~h}$ prior testing. The assays were performed between 2:00 and 4:00 pm. During the test, each animal was gently placed on the central platform, facing an open arm and with its back to the experimenter. A camera was mounted vertically (1.75 $\mathrm{m}$ ) above the arena and the videotape was analyzed by an observer who was blind to the prenatal treatment. The apparatus was cleaned with a $20 \%$-ethanol solution and dried with a cloth between sessions.

Changes in the percentage of time spent in the open arms indicate changes in anxiety-like behavior. The time spent in open arms, expressed as a percentage of total time spent in both open and closed arms, provides a measure of fearinduced inhibition of exploratory activity (27). We calculated the percentage of entries into the open arms (number of entries into the open arms/total number of arm entries $x 100$ ) and percentage of time spent in the open arms [time spent in the open arms/total time spent in arms $x$ 100].

Sexual behaviortest. At PN105, each male was allowed to have a previous experience with a sexually receptive female during a period of $24 \mathrm{~h}$. These animals were then evaluated in a copulatory test under dim red light illumination at PN120. The assays were performed in the evening (7:00 pm). Each rat was placed in a $25 \times 40 \times 38-\mathrm{cm}$ chamber and allowed a habituation period of $5 \mathrm{~min}$ after which a sexually receptive ovariectomized female (3-4 months old) was introduced into the chamber. The estrous phase was induced with estradiol benzoate $(20 \mu \mathrm{g} / \mathrm{kg}, s c)$ and progesterone $(100 \mathrm{mg} / \mathrm{mL}, s c)$ 24 and $6 \mathrm{~h}$, respectively, before the test.

During a single 30-min recorded copulatory test, the mounting, intromission and ejaculation latencies were computed as the time from the beginning of the experiment until the first response. The number of mountings, intromissions and ejaculations performed for 30 min was also computed. Every male was tested three times with a 1-week interval between measurements (28). A mounting is computed when the male approaches the female from the rear sometimes posing his forelegs over the female's back and making rapid anteroposterior pelvis thrusts. An intromission involves vaginal penetration; the male starts the mounting but suddenly makes a deep thrust forward, stops pelvic thrusting, withdraws vigorously, and licks his genitals. Finally, an ejaculation is considered to be a behavior starting as an intromission, but with the male remaining on the female for 1-3 s (28) after vaginal penetration. When these behavioral components did not occur the male was considered to be sexually inactive.

\section{Statistical analysis}

We used one-way analysis of variance (ANOVA) followed by the LSD Fisher test for multiple comparisons and cosinor methods (29) for statistical analysis of time series. Time determination data are given as the mean $\mathrm{U} / \mathrm{g}$ wet tissue \pm standard deviation (SD). Data were analyzed using a computer program for the best adjustment of the cosine regression model. We calculated the correlation coefficient ( $r$ ), a measure of the curve adjusted to the experimental data. A circadian rhythm was characterized by a) acrophase (time of the maximum value in fitted cosine function), b) amplitude (half the crest-trough difference of the fitted cosine function), and c) mesor (average value of the fitted cosine curve). Rhythm detection and its significance were determined by the zero-amplitude test (29) with $\mathrm{P}<0.05$. Mesor values are reported as the mean $\pm S D$. Data of elevated plus-maze and copulatory behavior were analyzed using the nonparametric Kruskal-Wallis test and the Mann-Whitney U-test. The Student $t$-test was used for unpaired data.

\section{Results}

The time of pregnancy, numbers of born pups and per- 
centage of male and female pups from mothers submitted to the different treatments were not affected. Although the body weight of offspring from the LL group was lower than that of the control group at 3 days of age (mean \pm SEM; control = 8.70 $\pm 0.17 \mathrm{~g}, \mathrm{~N}=9$; $\mathrm{LL}=7.29 \pm 0.20 \mathrm{~g}, \mathrm{~N}=9$; $\mathrm{P}<0.0001$, Student $t$-test), no difference was observed in the animals at 60 days of age (data not shown) or in pups with birth defects.

\section{Testicular circadian rhythms of the offspring}

Malate dehydrogenase. In 28-day-old pups born to undisturbed mothers, MDH activity showed a significant daily variation $\left(F_{8,36}=11.29, \mathrm{P}<0.0001\right.$, cosinor and ANOVA-LSD Fisher test), as shown in Figure 1A. The curve, adjusted to experimental values, was obtained by the cosinor method. The acrophase was found during the subjective night (circadian time $=16.00 \pm 0.75$ ). The results of cosinor analyses for circadian $\mathrm{MDH}$ rhythm are presented in Table 1.

When mothers were submitted to LL from the 10th to the 21st day of pregnancy (LL group), their pups did not show any significant daily variations in testicular $\mathrm{MDH}$ activity (Figure 1B). The mesor value was higher than that of control pups ( $P<0.05$, ANOVA and LSD Fisher test). The value of the amplitude was very low compared to the control group because there was no circadian rhythm $(\mathrm{P}>$ 0.05, ANOVA and LSD Fisher test, Table 1).

In contrast, when melatonin was administered to mothers submitted to constant illumination ( $L L+$ Mel group), the $\mathrm{MDH}$ postnatal circadian activity entrainment of their pups was preserved $\left(F_{7,39}=3.46, P<0.001\right.$, cosinor and ANOVA-
LSD Fisher test), as shown in Figure 1C. The mesor of this enzyme rhythm was lower than that of the animals of the LL group ( $P<0.05$, ANOVA and LSD Fisher test) and did not differ from the mesor of the control group. There was a significant difference in the amplitude between LL+Mel and control groups ( $P<0.05$, ANOVA and LSD Fisher test). The acrophase of the MDH rhythm of pups from the LL+Mel group occurred during the subjective day (circadian time $=$ $6.22 \pm 2.06)$ and was significantly different from that of the control group ( $\mathrm{P}<0.05$, Student $t$-test, Table 1$)$.

Lactate dehydrogenase. LDH activity showed a significant daily variation $\left(F_{8,37}=10.15, \mathrm{P}<0.0001\right.$, cosinor and ANOVA-LSD Fisher test) in pups born to control mothers, as shown in Figure 2A. This daily oscillation had an acrophase at the end of the subjective day (circadian time $=11.54 \pm$ 1.13). The results of cosinor analyses for circadian LDH rhythm are presented in Table 1.

When mothers were submitted to constant light from the 10th to the 21st day of pregnancy (LL group), their pups did not show a significant daily variation in testicular LDH activity (Figure 2B). The mesor value did not differ from that of the control group ( $P>0.05$, ANOVA and LSD Fisher test). The amplitude value was very low since there was no circadian rhythm (Table 1).

In contrast, when melatonin was administered to mothers submitted to constant illumination ( $L L+$ Mel group), the LDH postnatal circadian activity entrainment of their pups was preserved $\left(F_{8,37}=4.69, \mathrm{P}<0.001\right.$, cosinor and ANOVALSD Fisher test) in pups' testes (Figure 2C). The mesor of this daily oscillation was higher than that of pups from the LL group ( $\mathrm{P}<0.01$, ANOVA and LSD Fisher test). There was

Table 1. Circadian variables (cosinor) of testicular malate dehydrogenase and lactate dehydrogenase in 28-day-old male rats.

\begin{tabular}{|c|c|c|c|c|c|}
\hline Groups $^{a}$ & Rhythmb & Mesorc & Amplitude $^{c}$ & Acrophase $^{d}$ & $r^{e}$ \\
\hline \multicolumn{6}{|l|}{$\mathrm{MDH}$} \\
\hline Control & $P<0.05$ & $412.18 \pm 6.93(45)$ & $42.94 \pm 9.99$ & $16.00 \pm 0.75$ & 0.88 \\
\hline LL & $P>0.05$ & $431.34 \pm 4.72^{*}(62)$ & $9.8 \pm 6.5^{*}$ & - & 0.75 \\
\hline $\mathrm{LL}+\mathrm{Mel}$ & $P<0.05$ & $400.80 \pm 8.17(47)$ & $20.46 \pm 0.48^{\#}$ & $6.22 \pm 2.06^{+}$ & 0.87 \\
\hline \multicolumn{6}{|l|}{ LDH } \\
\hline Control & $P<0.05$ & $146.55 \pm 4.21(46)$ & $20.61 \pm 5.99$ & $11.54 \pm 1.13$ & 0.88 \\
\hline LL & $P>0.05$ & $142.14 \pm 1.39^{\circ}(63)$ & $1.76 \pm 1.77^{*}$ & - & 0.69 \\
\hline $\mathrm{LL}+\mathrm{Mel}$ & $P<0.05$ & $155.07 \pm 3.20(50)$ & $14.31 \pm 4.46$ & $5.92 \pm 1.19^{+}$ & 0.84 \\
\hline
\end{tabular}

aControl: pups born to mothers submitted to a schedule of 12-h light:12-h dark and injected subcutaneously with a daily dose of ethanol/saline solution from the 17 th to the 21 st day of pregnancy, at circadian time 12 (CT12). LL: pups born to mothers submitted to constant light from the 10th to 21 st day of gestation and injected subcutaneously with a daily dose of ethanol/saline solution from the 17th to 21 st day of pregnancy, at CT12. LL+Mel: pups born to mothers submitted to constant light as described for the LL group and treated daily with melatonin $(1 \mathrm{mg} / \mathrm{kg}, s c)$ from the 17 th to $21 \mathrm{st}$ day of pregnancy, at CT12. ${ }^{\mathrm{b}} \mathrm{A}$ circadian rhythm was present when the amplitude of variation was significantly different from zero $(P<0.05$, cosinor method). "Values are mean U/g tissue \pm SD. The number of animals is given in parentheses. dValues are mean $\mathrm{CT} \pm \mathrm{SD}$. e Correlation coefficient. ${ }^{*} \mathrm{P}<0.05$ compared to control and $\mathrm{LL}+\mathrm{Mel}$ groups, ${ }^{\#} \mathrm{P}<0.05$ compared to control and LL groups, ${ }^{\circ} \mathrm{P}<0.01$ compared to LL+Mel group (ANOVA followed by the LSD Fisher test), ${ }^{+} \mathrm{P}<0.01$ compared to control (Student $t$-test). 
no significant difference in the amplitude values between control and LL+Mel groups. The acrophase of this rhythm was observed during the subjective day (circadian time = $5.92 \pm 1.19)$ and was significantly different from that of the control group $(P<0.05$, Student $t$-test, Table 1$)$.
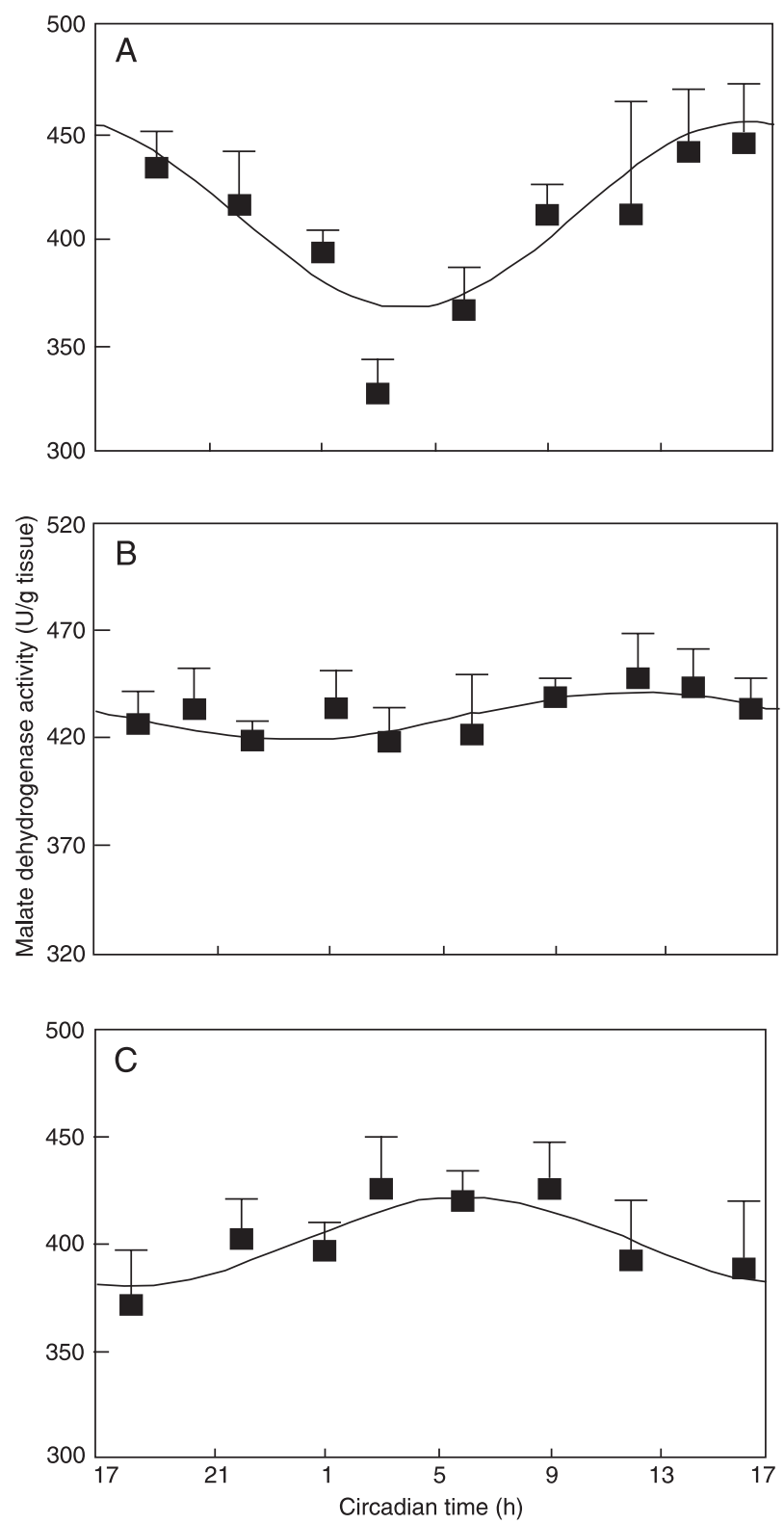

Figure 1. Daily malate dehydrogenase activity of testes from 28-dayold rats. Pups born to $A$, control group: enzymatic activity values at circadian time 14 (CT14), CT16 and CT19 were significantly higher than those at CT1, CT3 and CT6 $(P<0.05$, cosinor and ANOVA-LSD Fisher test); $B$, LL group; $C$, LL+Mel group: values at CT3 and CT9 were significantly higher than those at CT16 and CT18 $(P<0.05$, cosinor and ANOVA-LSD Fisher test). The time of activity onset under dark conditions was defined as CT12. The experimental values reported for each hour are means \pm SD of 5 to 8 animals.

\section{Long-term recording of anxiety-like and sexual behavior}

Anxiety-like behavior. The effectiveness of the stress induced by constant light during gestation was assessed in male offspring in the elevated plus-maze test. The animals
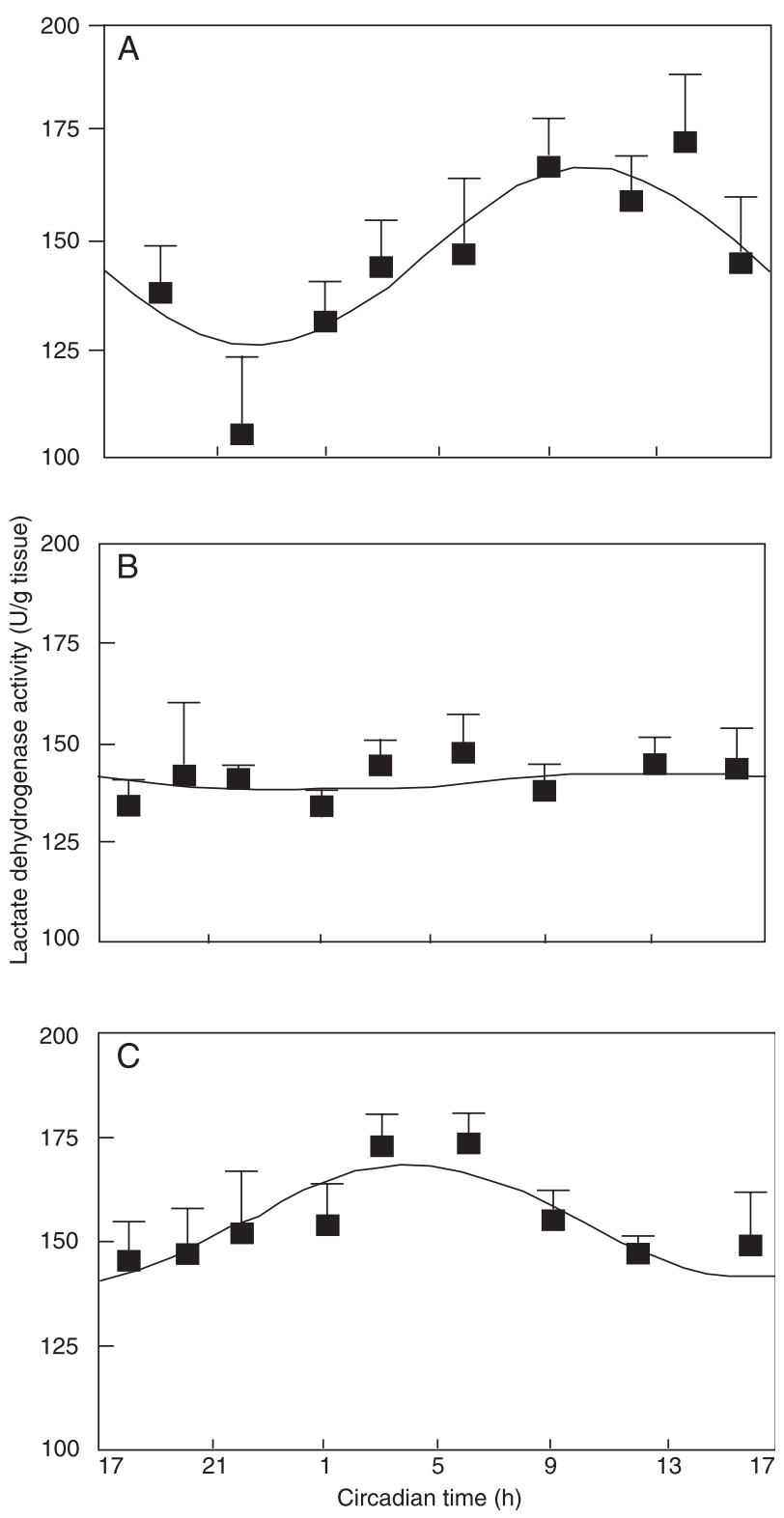

Figure 2. Daily lactate dehydrogenase activity of testes from 28-day-old rats. Pups born to $A$, control group: enzymatic activity values at circadian time 9 (CT9), CT12 and CT14 were significantly higher than those at CT1, CT19 and CT22 $(P<0.01$, cosinor and ANOVA-LSD Fisher test); $B$, LL group; $C$, LL+Mel group: the highest values were recorded at CT3 and CT6 $(P<0.01$, cosinor and ANOVA-LSD Fisher test). The time of activity onset under dark conditions was defined as CT12. The experimental values reported for each hour are means \pm SD for 5 to 8 animals. 
showed an alteration in the anxiety-like behavior when compared to the male control group (Figure 3). The percentage of entries into the open arms showed that prenatally LL rats entered the open arms significantly less often than control rats $(P<0.001$, Kruskal-Wallis test and Mann-Whitney $U$ test) and LL+Mel rats $(P<0.001$, Kruskal-Wallis test and Mann-Whitney U-test). There was no difference between the LL+Mel-treated group and the control group.

When the percentage of time spent in the open arms was considered, animals of the LL group spent significantly less time in the open arms than control animals $(P<0.001$, Kruskal-Wallis test and Mann-Whitney U-test) and LL+Mel rats $(P<0.002$, Kruskal-Wallis test and Mann-Whitney $U$-test). There was no difference between the prenatally LL+Mel-treated animals and the control group.

Sexual behavior. The sexual behavior of the male control pups was significantly different from that of prenatal LL adult animals. The LL male offspring presented higher values of mounting, intromission and ejaculation latencies (Figure 4), and lower numbers of these events (Figure 5) compared to control $(P<0.01$, Kruskal-Wallis test and Mann-Whitney U-test).

Interestingly, maternal melatonin treatment protected pups from the adverse effects of LL. In the LL+Mel group, the mounting, intromission and ejaculation latency episodes and number of these events were similar to the values of the control group (Figures 4 and 5). There was no significant difference between control and LL+Mel animals in any of the parameters recorded.

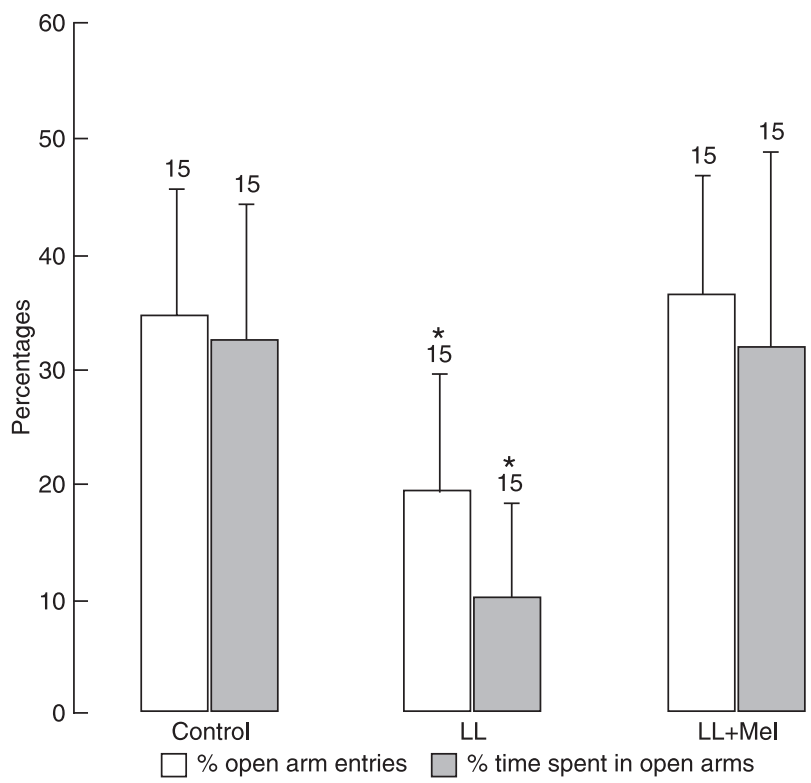

Figure 3. Long-term effect of prenatal treatment on anxiety-like behavior at postnatal day 60 (PN60). Data are reported as means $\pm \mathrm{SD}$. The number of rats is given on the top of each bar. ${ }^{*} \mathrm{P}<$ 0.001 for the LL group vs control and LL+Mel groups (KruskalWallis test and Mann-Whitney U-test).

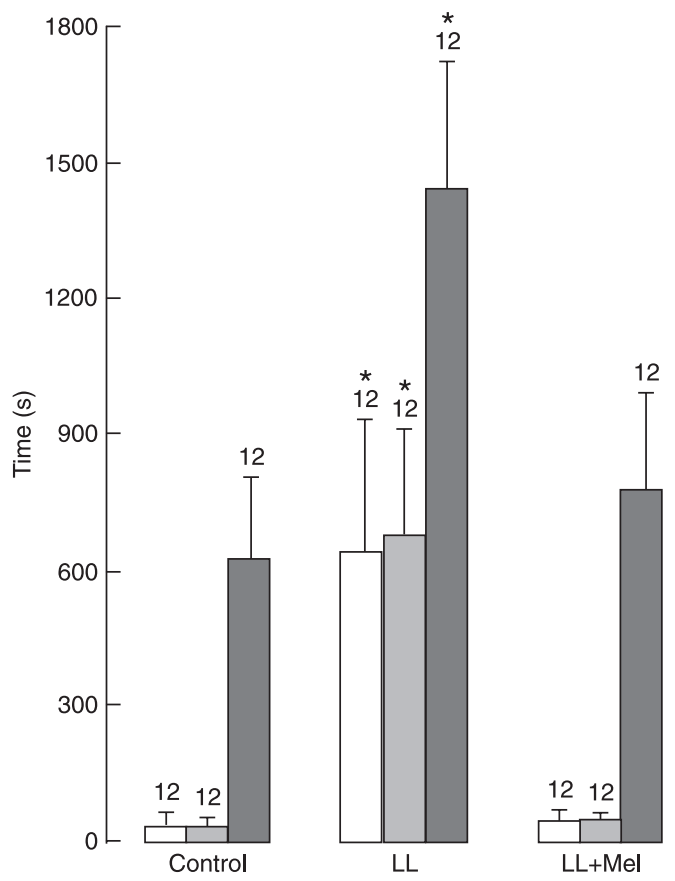

$\square$ Mount latency

Intromission latency

Ejaculation latency

Figure 4. Long-term effect of prenatal treatment on mounting, intromission and ejaculation latencies of sexual behavior at postnatal day 120 (PN120). Data are reported as means \pm SD. The number of rats is given on the top of each bar. ${ }^{*} P<0.01$ for the LL group vs control and LL+Mel groups (Kruskal-Wallis test and Mann-Whitney U-test).

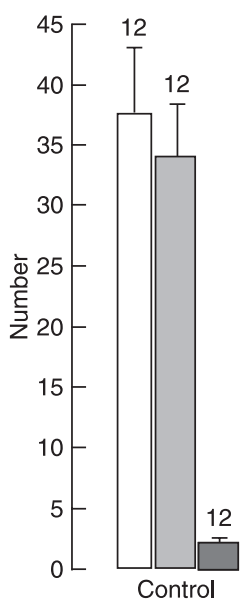

$\square$ Mount number

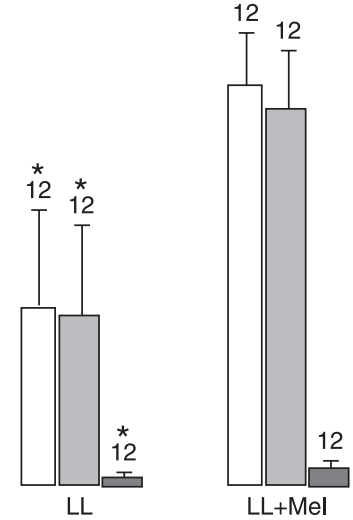

Intromission number $\square$ Ejaculation numbe।
Figure 5. Long-term effect of prenatal treatment on mounting, intromission and ejaculation numbers in the sexual behavior at postnatal day 120 (PN120). Data are reported as means \pm SD. The number of rats is on the top of each bar. ${ }^{*} \mathrm{P}<0.01$ for the LL group vs control and LL+Mel groups (Kruskal-Wallis test and Mann-Whitney U-test). 


\section{Discussion}

The present study showed, for the first time, that constant light during gestation induces an early disruption of daily oscillation of MDH and LDH in offspring rat testes together with long-term effects on anxiogenic and copulatory behavior patterns. These effects of LL on the maternal SCN were prevented by maternal melatonin administration during the last period of gestation. Our results are consistent with previous studies $(6,7,11,12,30)$, confirm the importance of the melatonin signal and provide evidence of the protective effects of the hormone on maternal programming during gestation.

\section{Circadian rhythms of testicular enzymes in the offspring}

Several studies on maternal entrainment have shown that during embryonic and early neonatal development the circadian rhythms of the fetuses develop in synchrony with those of their mothers, which are synchronized with the environmental photoperiod $(14,15,17)$. Since there are no neural connections between pregnant rats and fetuses, this synchronization must be mediated by maternal signal(s) reaching the fetuses through the placental circulation $(5,17)$ or during the lactation period $(7,14)$. In the present study, we showed the presence of a significant daily oscillation of MDH and LDH activities at PN28 in the testes of control pups kept in constant darkness from birth (Figures $1 \mathrm{~A}$ and $2 \mathrm{~A}$ ). Their phase distribution probably was clustered around the mother's circadian phase, as was observed for drinking behavior (12). We demonstrated that circadian phase distributions for the drinking circadian behavior of individual pups born to control mothers and kept in constant darkness from birth were significantly different from uniform distribution and clustered near those of the mother's circadian phase (12).

LL exposure between the 10th and 21st days of gestation probably disrupted the transfer of the maternal signal(s) and consequently the rhythms of $\mathrm{MDH}$ and $\mathrm{LDH}$ activities in male offspring (Figures 1B and 2B). This absence of enzymatic rhythms might mean that each individual pup lacks a circadian rhythm or that the phases vary significantly, so that the enzymatic activity average would appear to be arrhythmic. Interestingly, these results are similar to those obtained after maternal removal of both superior cervical ganglia before day 11 of pregnancy. In effect, rat pups whose mothers were ganglionectomized by extirpation of the superior cervical ganglia, did not have a testicular MDH rhythm (7) and showed a significant dispersion in drinking circadian phases, within and among litters, monitored in individual animals starting after weaning and studied up to 3 weeks later (12).

Thus, the constant prenatal light could generate the same effect of maternal ganglionectomy on pups' testicular enzyme entrainment, that is, a phase shift between their individual profiles. As a consequence, the absence of amplitude in the adjusted curve of pups' MDH and LDH activities after prenatal LL treatment could be interpreted as an inter-individual desynchronization of pups' SCN, since the fetal clock entrainment would depend on the oscillations of synchronized neurons of the maternal clock, the SCN (18). Constant light has been shown to disrupt the circadian organization in adult rodents due to an individual desynchronization of clock neurons (31). These data agree with those obtained in studies on fetuses of capuchin monkeys from mothers exposed to constant light, which showed a significant alteration of fetal expression of the SCN clock genes Bmal-1 and melatonin-1 receptor (32). Thus, the disruption of pup circadian organization would explain the alteration of mother clock ability to generate circadian signal(s) under adverse effects of prenatal LL. It is tempting to speculate that the expression of SCN during postnatal life is a consequence of prenatal desynchronization of the fetal SCN.

\section{Long-term recording of anxiety-like and sexual behavior}

Constant light applied to pregnant rat causes long-term influences on offspring behavior during postnatal life. The results of the plus-maze test showed that male offspring of the LL group entered and spent significantly less time in the open arms, suggesting a more anxious behavior compared to offspring of control mothers (Figure 3). Probably, an anxiogenic effect of constant light on pregnant rats might result in several types of fetal development disruption.

Several investigators have applied different stressful models to pregnant rats and have obtained different anxietylike responses $(20,33)$. Those studies did not correlate with our observations because of the different nature and frequency of adverse intrauterine environment conditions endured by mothers during pregnancy.

Our findings about the copulatory behavior of adult male offspring born to mothers exposed to LL showed a delay in mounting, intromission and ejaculation latency values, as well as a decrease in the number of these events (Figures 4 and 5). Indeed, our data are similar to those obtained by chronic prenatal restraint stress on anxiety-like and copulatory behavior (24). It is known that maternal stress generates an oxidative status in mother and neonate (34), and decreases the surge of testosterone on gestational days 18 and 19 in male fetuses, a period postulated to be critical for the development of the central nervous system in rats (35). In summary, it is possible that the behavioral dysfunctions caused by unfavorable conditions generated by constant light were similar to those observed in chronic stress during gestation.

\section{Melatonin administration to the mothers during gestation}

The available literature on maternal entrainment in- 
dicates that there might be a number of maternal signals responsible for pup synchronization, and the most important one seems to be melatonin (36). Melatonin has been proposed as an efferent hormonal output of the circadian clock, since pineal hormone can modulate the clock function through direct action on hormone receptors in the $\operatorname{SCN}(1,9)$.

The presence of an acrophase in the significant daily variation of $\mathrm{MDH}$ and $\mathrm{LDH}$ in testes of rats born to LL mothers treated with melatonin during the last period of pregnancy supports the involvement of the maternal pineal gland in the entrainment of the enzyme circadian rhythms (Figures $1 \mathrm{C}$ and $2 \mathrm{C}$ ). However, the lower $\mathrm{MDH}$ amplitude and the shift of acrophase observed in these rhythms may be due to a different blood concentration of melatonin and clock setting between the physiological melatonin increase in control mothers and the temporal maintenance of the exogenous melatonin increase generated by injection of the hormone in mothers of the LL+Mel group during the subjective dark period. Thus, a melatonin rhythm would be reestablished in mothers, resulting in a protective effect on testicular enzyme rhythms of their pups at PN28 (Figures 1C and 2C), anxiety-like behavior at PN60 (Figure 3) and sexual behavior at PN120 (Figures 4 and 5). There is a remarkable increase of pups' copulatory indexes after systemic injection of melatonin to mothers despite the fact that melatonin in photoperiodic rodent species is usually an inhibitory signal

\section{References}

1. Hardeland R. Melatonin, hormone of darkness and more: occurrence, control mechanisms, actions and bioactive metabolites. Cell Mol Life Sci 2008; 65: 2001-2018.

2. Tan DX, Reiter RJ, Manchester LC, Yan MT, El-Sawi M, Sainz RM, et al. Chemical and physical properties and potential mechanisms: melatonin as a broad spectrum antioxidant and free radical scavenger. Curr Top Med Chem 2002; 2: 181-197.

3. Persengiev SP. The neuroprotective and antiapoptotic effects of melatonin in cerebellar neurons involve glucocorticoid receptor and p130 signal pathways. J Steroid Biochem Mol Biol 2001; 77: 151-158.

4. Baydas G, Reiter RJ, Nedzvetskii VS, Nerush PA, Kirichenko SV. Altered glial fibrillary acidic protein content and its degradation in the hippocampus, cortex and cerebellum of rats exposed to constant light: reversal by melatonin. J Pineal Res 2002; 33: 134-139.

5. Klein DC. Evidence for the placental transfer of $3 \mathrm{H}$-acetylmelatonin. Nat New Biol 1972; 237: 117-118.

6. Davis FC, Mannion J. Entrainment of hamster pup circadian rhythms by prenatal melatonin injections to the mother. Am J Physiol 1988; 255: R439-R448.

7. Vermouth NT, Carriazo CS, Gallara RV, Carpentieri AR, Bellavia SL. Maternal coordination of the daily rhythm of malate dehydrogenase activity in testes from young rats: effect of maternal sympathetic denervation of the pineal gland and administration of melatonin. Chronobiol Int 1995; 12: 8-18. for reproduction $(37,38)$. This result suggests an entrainment effect of melatonin during pregnancy rather than an action of the hormone on the reproductive tract per se. We have also found that melatonin injection in pinealectomized or ganglionectomized mothers on the 7th day of gestation prevents, within and among litters, the disruption of the individual circadian rhythm of pup drinking behavior in free running period, phase and amplitude (12).

Our finding of the protective action of melatonin on maternal programming during gestation is relevant. It could be related to entrainment property, favoring the neural coupling of the fetal multioscillatory system of all metabolic and behavioral processes in parallel. However, the maternal entrainment under circadian control may be the result of a more complex mechanism rather than in response to a single hormonal output.

\section{Acknowledgments}

Research supported, in part, by grants from CONICET (National Council of Scientific and Technological Research) and from SECYT (Secretaría de Ciencia y Técnica) of the National University of Córdoba, Argentina. We thank Dr. Otto Orsingher of the Universidad Nacional de Córdoba, Argentina, for helpful comments and excellent technical assistance. N.T. Vermouth is a Career Investigator of CONICET.
8. Tamura H, Takayama H, Nakamura Y, Reiter RJ, Sugino N. Fetal/placental regulation of maternal melatonin in rats. $J$ Pineal Res 2008; 44: 335-340.

9. Williams LM, Martinoli MG, Titchener LT, Pelletier G. The ontogeny of central melatonin binding sites in the rat. Endocrinology 1991; 128: 2083-2090.

10. Seron-Ferre M, Ducsay CA, Valenzuela GJ. Circadian rhythms during pregnancy. Endocr Rev 1993; 14: 594-609.

11. Bellavía SL, Carpentieri AR, Vermouth NT. Prenatal entrainment of rat testicular malate dehydrogenase activity circadian rhythm. Biol Rhythm Res 1996; 27: 302-313.

12. Bellavia SL, Carpentieri AR, Vaque AM, Macchione AF, Vermouth NT. Pup circadian rhythm entrainment - effect of maternal ganglionectomy or pinealectomy. Physiol Behav 2006; 89: 342-349.

13. Reppert SM, Weaver DR. Coordination of circadian timing in mammals. Nature 2002; 418: 935-941.

14. Deguchi T. Ontogenesis of a biological clock for serotonin:acetyl coenzyme A N-acetyltransferase in pineal gland of rat. Proc Natl Acad Sci U S A 1975; 72: 2814-2818.

15. Reppert SM. Interaction between the circadian clocks of mother and fetus. Ciba Found Symp 1995; 183: 198-207.

16. Davis FC, Gorski RA. Entrainment of circadian rhythms in utero: role of the maternal suprachiasmatic nucleus. Soc Neurosci 983; 9: 625.

17. Reppert SM, Schwartz WJ. Maternal coordination of the fetal biological clock in utero. Science 1983; 220: 969-971. 
18. Reppert SM, Schwartz WJ. Maternal suprachiasmatic nuclei are necessary for maternal coordination of the developing circadian system. J Neurosci 1986; 6: 2724-2729.

19. Depres-Brummer P, Levi F, Metzger G, Touitou Y. Lightinduced suppression of the rat circadian system. Am J Physiol 1995; 268: R1111-R1116.

20. Weinstock M. Alterations induced by gestational stress in brain morphology and behaviour of the offspring. Prog Neurobiol 2001; 65: 427-451.

21. Vermouth NT, Ponce RH, Carriazo CS, Blanco A. Circadian rhythm of lactate dehydrogenase in rat testis. Comp Biochem Physiol B 1984; 78: 897-902.

22. Carriazo CS, Ponce RH, Sereno R, Vermouth NT. Malate dehydrogenase activity of rat testis: circadian rhythm and its ontogeny. Biol Rhythm Res 1998; 29: 300-310.

23. Estanislau C, Morato S. Prenatal stress produces more behavioral alterations than maternal separation in the elevated plus-maze and in the elevated T-maze. Behav Brain Res 2005; 163: 70-77.

24. Gerardin DC, Pereira OC, Kempinas WG, Florio JC, Moreira EG, Bernardi MM. Sexual behavior, neuroendocrine, and neurochemical aspects in male rats exposed prenatally to stress. Physiol Behav 2005; 84: 97-104.

25. Ochoa S. Malic dehydrogenase from pig heart. In: Colowick SP, Kaplan NO (Editors), Methods in enzymology. New York: Academic Press; 1955. p 735-736.

26. Wroblesky F, LaDue JS. Lactic dehydrogenase activity in blood. Proc Soc Exp Biol Med 1955; 90: 210-213.

27. Pellow S, Chopin P, File SE, Briley M. Validation of open:closed arm entries in an elevated plus-maze as a measure of anxiety in the rat. $J$ Neurosci Methods 1985; 14 : 149-167.

28. Agmo A. Male rat sexual behavior. Brain Res Brain Res Protoc 1997; 1: 203-209.

29. Nelson W, Tong YL, Lee JK, Halberg F. Methods for cosinor- rhythmometry. Chronobiologia 1979; 6: 305-323.

30. Warren WS, Cassone VM. The pineal gland: photoreception and coupling of behavioral, metabolic, and cardiovascular circadian outputs. J Biol Rhythms 1995; 10: 64-79.

31. Ohta H, Yamazaki S, McMahon DG. Constant light desynchronizes mammalian clock neurons. Nat Neurosci 2005; 8: 267-269.

32. Torres-Farfan C, Rocco V, Monso C, Valenzuela FJ, Campino C, Germain A, et al. Maternal melatonin effects on clock gene expression in a nonhuman primate fetus. Endocrinology 2006; 147: 4618-4626.

33. Richardson HN, Zorrilla EP, Mandyam CD, Rivier CL. Exposure to repetitive versus varied stress during prenatal development generates two distinct anxiogenic and neuroendocrine profiles in adulthood. Endocrinology 2006; 147: 2506-2517.

34. Arguelles S, Machado MJ, Ayala A, Machado A, Hervias B. Correlation between circulating biomarkers of oxidative stress of maternal and umbilical cord blood at birth. Free Radic Res 2006; 40: 565-570.

35. Ward IL, Weisz J. Differential effects of maternal stress on circulating levels of corticosterone, progesterone, and testosterone in male and female rat fetuses and their mothers. Endocrinology 1984; 114: 1635-1644.

36. Redman JR, Armstrong SM, Ng KT. Free-running activity rhythms in the rat: entrainment by melatonin. Science 1983; 219: 1089-1091.

37. Vanecek J. Inhibitory effect of melatonin on $\mathrm{GnRH}$-induced LH release. Rev Reprod 1999; 4: 67-72.

38. Frungieri MB, Mayerhofer A, Zitta K, Pignataro OP, Calandra RS, Gonzalez-Calvar SI. Direct effect of melatonin on Syrian hamster testes: melatonin subtype 1a receptors, inhibition of androgen production, and interaction with the local corticotropin-releasing hormone system. Endocrinology 2005; 146: 1541-1552. 